\title{
\begin{tabular}{c} 
TERAPIA \\
PSICOLÓGICA \\
\hline ISSN: 0718-4808
\end{tabular}

\author{
Garikoitz Mendigutxia-Sorabilla \\ (iD) 0000-0003-2113-3905 \\ gmendigutxia@outlook.com \\ Fundación Proyecto Hombre de Navarra. \\ Programa Suspertu. \\ Pamplona (Iruña), España.
}

\section{Comportamientos de riesgo y dimensiones de personalidad en la adolescencia}

\section{Risk behaviours and personality traits in adolescence}

\section{Resumen:}

Los objetivos del presente trabajo fueron: 1) establecer la prevalencia del consumo de alcohol, de tabaco y de cannabis, así como de las relaciones sexuales sin protección y la repetición de curso en adolescentes en función del sexo; 2) determinar las dimensiones de personalidad introversión (I), búsqueda de sensaciones (BS), impulsividad (Im) y sensibilidad a la ansiedad (SA) en función del sexo; 3) relacionar posibles perfiles de personalidad con dichas conductas. La muestra se compuso de 1.324 adolescentes (14-18 años). A partir de las dimensiones de personalidad valoradas se establecieron 4 grupos de adolescentes. El grupo de adolescentes con puntuaciones altas en todas las dimensiones de personalidad es el que presentó mayores tasas de experimentación con el consumo. Por el contrario, el de los adolescentes con bajos niveles de I, BS e Im fue el que menores tasas presentó. Las intervenciones preventivas deberían tener en cuenta las dimensiones de personalidad señaladas para poder aplicarse con una mayor efectividad.

Palabras Clave: personalidad; adolescencia; comportamientos de riesgo; prevención.

\section{Abstract:}

The objectives of this study were: 1) to establish the prevalence of alcohol, tobacco and cannabis use, as well as unprotected sex and school failure among adolescents according to gender; 2) to determine the personality dimensions: introversion (I), sensation-seeking (SS), impulsivity (Im) and anxiety sensitivity (AS) according to gender; 3) to relate possible personality profiles to these behaviours. The sample included 1.324 adolescents aged between 14-18. 4 groups of adolescents were established based on the assessed personality dimensions. The group of adolescents with high scores in all personality dimensions presented the highest rates of experimentation with consumption. On the contrary, the group of adolescents with low levels of I, SS and Im presented the lowest rates. Preventive interventions should take into account the personality dimensions indicated in order to be applied with greater effectiveness.

Keywords: personality; adolescence; risk behaviours; prevention. 


\section{Introducción}

La adolescencia es una etapa clave en el inicio de diferentes comportamientos que pueden suponer consecuencias negativas para la salud, tanto a corto, medio como a largo plazo. Entre ellas destacan el consumo de drogas o las relaciones sexuales de riesgo. La encuesta estatal realizada por la Delegación del Gobierno para el Plan Nacional sobre Drogas sobre el uso de drogas en estudiantes de enseñanza secundaria, indicaba que el $77,9 \%$ de los estudiantes de 14 a 18 años habían probado el alcohol, el 41,3\% el tabaco y el $33 \%$ el cánnabis. La edad media de inicio en el consumo del alcohol se situaba en los 14 años, en los 14,1 en el caso del tabaco y en los 14,9 en el cánnabis. Por sexo, las mujeres habían probado más alcohol $(79,4 \%$ vs. $76,3 \%)$ y tabaco $(44,3 \%$ vs. $38,2 \%)$ y los hombres más cánnabis $(34,5 \%$ vs. $31,5 \%$ ) (Observatorio Español de las Drogas y las Adicciones, 2020). En cuanto a las relaciones sexuales, un $23,6 \%$ de los encuestados en una muestra de 1200 jóvenes con edades comprendidas entre los 16 y los 25 años a nivel nacional, manifiestan no utilizar siempre métodos anticonceptivos (Observatorio de Salud Sexual y Reproductiva de la Sociedad Española de Contracepción, 2019). En Navarra un 12,8 \% de los chicos y chicas encuestados entre los 14 y los 17 años, reconocieron no haber utilizado ningún tipo de protección en su primera relación sexual, no encontrándose diferencias entre hombres y mujeres (González-Eransus \& Miqueléiz, 2016).

Se han relacionado diferentes variables del ámbito escolar con el consumo de drogas, entre las que destacan el absentismo, el bajo rendimiento, los problemas de comportamiento en el aula y la repetición de curso (Pinazo, Diez, \& Rouma, 2002). De hecho, la repetición de algún curso escolar correlaciona positivamente con el fracaso escolar (Martín, 2007) y tiene efectos negativos a diferentes niveles, entre ellos el aumento de problemas de conducta (Farías, Fiol, Kit, \& Melgar, 2007; López-Larrosa \& Rodríguez-Arias, 2012; Lucchese, Burrone, Enders, y Fernández, 2013). Éste es un dato importante, puesto que según datos del informe PISA de 2018, en España tomado en su conjunto, el 28,7\% del alumnado español había repetido alguna vez, 16 puntos porcentuales mayor que en el total de la Unión Europea. En cuanto al sexo, el porcentaje de hombres que repiten es mayor que el de las mujeres. En Navarra, este porcentaje es menor $(23,89 \%)$ que la media nacional, pero 11 puntos superior al promedio de la OCDE (Ministerio de Educación y Formación Profesional, 2019).

Para algunos autores los consumos de sustancias responden a una forma de superar o afrontar determinados estados emocionales. Se ha observado que aquellas personas que presentan problemas en la regulación de emociones tales como la ansiedad, son más 
proclives al consumo de drogas. El consumo de drogas está relacionado con los problemas de inadaptación personal a la sociedad, debidos a un manejo inadecuado de la ansiedad, las frustraciones, las inquietudes o protestas, entre otros (Cano-Vindel, Miguel-Tobal, González, \& Iruarrizaga, 1994) o como modo de afrontamiento del dolor y la tristeza (Croese \& van Hoof, 2020). También se han relacionado los estados de ánimo depresivos con el consumo de sustancias. En una muestra de 1507 adolescentes españoles, los que eran fumadores presentaban un peor estado de ánimo que los no fumadores y además consumían con mayor intensidad. Resultaba más probable ser fumador en presencia de un estado de ánimo bajo (Gonzálvez, Espada, \& Orgilés, 2015). También se ha encontrado que estudiantes con niveles más altos de síntomas depresivos eran más propensos a usar cannabis en diferentes situaciones (Beck et al., 2009). En este sentido se ha propuesto que las iniciativas antitabáquicas dirigidas a los jóvenes deben considerar los problemas de malestar emocional y el consumo de tabaco como una forma de automedicación (Carceller-Maicas et al., 2015).

Se ha relacionado el inicio de los consumos de drogas en la adolescencia con una dimensión de personalidad denominada búsqueda de sensaciones. Ésta se ha definido habitualmente, por una baja tolerancia al aburrimiento, una fuerte necesidad de estimulación, y una decidida intención de asumir riesgos y experiencias variadas (Arnett, 1994; Pérez de Albéniz-Garrote, \& Medina, 2019; Zuckerman, 1979). En una muestra de adolescentes en Israel, se encontró que cuando había disponibilidad de drogas, los adolescentes caracterizados por una búsqueda de sensaciones alta, consumían más drogas que los que no tenían una puntuación alta en esa dimensión. Sin embargo, no se encontró relación entre el estado de ánimo y el consumo (Teichman, Barnea, \& Rahav, 1989). Estos resultados se han visto corroborados más recientemente en una revisión meta-analítica con respecto a los consumos de alcohol en adolescentes. El consumo excesivo de alcohol durante la adolescencia podría estar impulsado en parte, por el deseo de buscar experiencias nuevas y emocionantes (Stautz \& Cooper, 2013).

La impulsividad es otra dimensión de personalidad que se ha estudiado en relación con las conductas de riesgo. Se define como la tendencia a realizar determinadas acciones con escasa previsión y sin considerar sus consecuencias (Adan, 2012). En una muestra de adolescentes en Inglaterra, se encontró relación entre la impulsividad y mayores consumos de alcohol (Fernie et al., 2013). En el mismo sentido, en una muestra de adolescentes de Almería (España), aquellos estudiantes que se declararon consumidores de alcohol y tabaco, obtuvieron puntuaciones significativamente más altas en impulsividad (Pérez-Fuentes et al., 2015). 
También se han encontrado relaciones significativas entre la impulsividad y la búsqueda de sensaciones con diferentes comportamientos de riesgo en los adolescentes, como los consumos de drogas o el juego problema (Leeman, Hoff, Krishnan-Sarin, Patock-Peckham, \& Potenza, 2014). En España se encontró que la búsqueda de sensaciones predecía un mayor consumo en adolescentes consumidores de alcohol y cannabis, mientras que la impulsividad predecía un mayor consumo de tabaco (Aleixandre, Pol, \& del Río, 2005).

La única herramienta de evaluación de la personalidad que mide los anteriores cuatro rasgos de personalidad de forma conjunta (desesperanza, búsqueda de sensaciones, impulsividad y sensibilidad a la ansiedad) y que es particularmente adecuada para la autoadministración en adolescentes y adultos se denomina Substance Use Risk Profile (SURPS; Woicik, Stewart, Pihl, \& Conrod, 2009). Con este instrumento, Malmberg et al. (2012) realizaron un estudio longitudinal con 758 adolescentes entre 11 y 14 años. En este estudio encontraron que la desesperanza y la búsqueda de sensaciones eran predictoras del consumo de tabaco y alcohol, y la búsqueda de sensaciones del cánnabis. A partir de un análisis de clases latentes, identificaron tres perfiles diferenciados en hombres y dos en mujeres. En contra de lo previsto por los autores, no hallaron diferencias en función de los diferentes perfiles (ni en hombres ni en mujeres) en el consumo de sustancias. Los autores concluyeron que el análisis por separado de las dimensiones de personalidad era más relevante en la predicción del inicio en el consumo que los perfiles de personalidad.

Hasta la fecha, no se han realizado en España investigaciones que relacionen las dimensiones de personalidad introversión, búsqueda de sensaciones, impulsividad y sensibilidad a la ansiedad, con los comportamientos de riesgo en la adolescencia. Descubrir cómo se relacionan estas variables con diferentes comportamientos de riesgo puede orientar en el diseño de estrategias de intervención más eficaces, en este caso, en el contexto educativo. Por lo tanto, los objetivos del presente trabajo son: 1) establecer la prevalencia del consumo de alcohol, de tabaco y de cannabis, así como de las relaciones sexuales sin protección y la repetición de curso en una muestra de adolescentes en función del sexo; 2) determinar las dimensiones de personalidad introversión, búsqueda de sensaciones, impulsividad y sensibilidad a la ansiedad, en una muestra de adolescentes en función del sexo; y 3) relacionar posibles perfiles de personalidad con las conductas valoradas.

\section{Métodos}

El proyecto cuenta con la autorización Pl-003/14 del Comité de Ética, Experimentación animal y Bioseguridad de la Universidad Pública de Navarra. Para la realización de la presente 
investigación se obtuvo, además, la autorización de los diferentes centros que formaron parte de la muestra. La dirección de cada uno de los centros se encargó de solicitar el permiso para la participación, la distribución de los cuestionarios y la recolección de los mismos. El alumnado rellenó el cuestionario dentro del horario escolar, y en todo momento se aseguró la confidencialidad de sus respuestas.

\section{Participantes}

La muestra se compuso de 1.324 adolescentes escolarizados en el año 2015 en siete centros escolares de la Comunidad Foral de Navarra. Semejantes en tamaño y procedencia geográfica del alumnado. Cuatro de los centros se situaban en Pamplona y su comarca, dos en la zona media y el último en la zona norte de Navarra. Cinco de los centros eran públicos (uno de ellos de Formación Profesional) y dos eran concertados (también uno de Formación Profesional), proporción que se asemeja al porcentaje de centros de titularidad pública $(75,6 \%)$ y privada $(24,4 \%)$ que imparten enseñanzas secundarias. De ellos, el $51,9 \%(n=687)$ eran hombres y el $48,1 \%$ mujeres $(n=637)$ de $2^{\circ}$ y $4^{\circ}$ de Educación Secundaria Obligatoria, $\left(2^{\circ}\right.$ Bachiller y $1^{\circ}$ y $2^{\circ}$ de Grados Medios) de, con edades comprendidas entre los 12 y los 19 años. La edad media era 15,1 (D.T. $=1,8$ ).

\section{Instrumentos}

Substance Use Risk Profile (SURPS). Éste es un cuestionario autoadministrado que identifica cuatro dimensiones de personalidad: 1) sensibilidad a la ansiedad; 2) desesperanza (introversión/desamparo/depresión); 3) búsqueda de sensaciones y 4) impulsividad. Está compuesto por 23 ítems, y cada dimensión se valora usando entre 5 y 7 ítems que se responden en una escala tipo Líkert de 1 (completamente en desacuerdo) a 5 (completamente de acuerdo). La sensibilidad a la ansiedad se describe como un miedo a la ansiedad relacionada con diferentes sensaciones físicas (Reiss, Peterson, Gursky, \& McNally, 1986). Un ejemplo es el ítem "Me asusta sentirme mareado o que me desmayo". En esta dimensión, con un rango 5-25, una puntuación alta indica una gran sensibilidad a la ansiedad. El segundo factor hace referencia a una tendencia hacia un estado de ánimo bajo, sentimientos de inutilidad y las creencias negativas sobre uno mismo, el mundo y el futuro (Castellanos \& Conrod, 2006; Conrod, Pihl, Stewart, \& Dongier, 2000). Un ejemplo de esta subescala es "Siento que soy un fracaso". En este caso, el rango es de 7-35, y una puntuación alta indica desamparo/depresión, mientras que una puntuación baja indica bienestar. La búsqueda de sensaciones se caracteriza por el deseo de intentar o probar cosas nuevas y está asociada con una elevada necesidad de estimulación e intolerancia al aburrimiento (Arnett, 1994; Woicik et al., 2009) (por ejemplo, "Me gusta hacer cosas que me asustan un poco"). El rango es de 
6-30, y una puntuación alta indica un gran deseo de intentar o probar experiencias nuevas. Por último, la impulsividad se caracteriza por una toma de decisiones rápidas y por respuestas inadecuadas con escasa previsión de las consecuencias (Castellanos-Ryan, Rubia, \& Conrod, 2011; Conrod, Castellanos-Ryan, \& Strang, 2010). Un ejemplo de esta dimensión es "Frecuentemente me meto en situaciones de las que me arrepiento después". En este caso, el rango es de 5-25, y una puntuación alta indica tendencia a la impulsividad.

Este instrumento es un buen predictor de conductas de riesgo, especialmente entre adolescentes. La estructura factorial, la consistencia interna y la fiabilidad test-retest, así como la validez de constructo, la validez convergente y la validez discriminativa se han mostrado adecuadas en adolescentes de diferentes países (Jurk et al., 2015; Krank et al., 2011; Lammers, Kuntsche, Engels, Wiers, \& Kleinjan, 2013; Newton et al., 2016; Robles-García et al., 2014).

Communities that Care Youth Survey (Arthur, Hawkins, Pollard, Catalano, \& Baglioni, 2002; Glaser, Horn, Arthur, Hawkins, \& Catalano, 2005). Este cuestionario permite identificar el consumo de drogas, la conducta antisocial, así como sus factores de riesgo y protección en la familia, la escuela, la comunidad, el propio individuo y sus iguales. Ha sido empleado con muestras españolas (López-Larrosa \& Rodríguez-Arias, 2012). Se contesta de forma anónima durante aproximadamente unos 45 minutos y es apropiado para adolescentes entre 11 y 18 años. El instrumento contempla tres criterios de sinceridad: inconsistencia, consumo inventado e insinceridad, que, si coinciden, excluyen al sujeto de la muestra. En este estudio se han empleado únicamente los 5 ítems del cuestionario referidos a comportamientos de riesgo (consumo tabaco, alcohol, cánnabis, relaciones sexuales sin protección y repetición de curso). Un ejemplo de uno de estos ítems es "¿Has fumado cigarrillos alguna vez en tu vida?"

\section{Procedimiento}

Tras la obtención de los permisos necesarios, se acudió en horario lectivo a los diferentes centros educativos, y tras una breve explicación en cada una de las aulas, el responsable del estudio distribuyó los cuestionarios entre el alumnado sin estar el profesorado presente. El tiempo medio de contestación del alumnado fue de 50 minutos.

Tras la obtención de los datos se mantuvieron reuniones con los centros educativos para comentar los resultados obtenidos y proponer una reflexión sobre las posibles medidas educativas a seguir. 


\section{Análisis de datos}

SURPS, se calcularon los percentiles de las mismas, tanto para el grupo total como para las submuestras de hombres y mujeres. Se realizó una comparación en las dimensiones de personalidad entre las personas que habían realizado algún comportamiento de riesgo (consumo de alcohol, tabaco, cánnabis y conductas sexuales de riesgo) o habían repetido curso. Como contraste de hipótesis se empleó la prueba de $t$ de Student para grupos independientes. Posteriormente, y a partir de las variables de personalidad de la muestra, se realizaron dos análisis de clúster para obtener tres y cuatro grupos de adolescentes. Con el propósito de maximizar la homogeneidad de cada uno de los grupos obtenidos se realizó el análisis de conglomerados de k-medias. Tras un análisis de los grupos obtenidos, se optó por escoger el de cuatro grupos ya que el alumnado se distribuía de una manera más equilibrada. Estos cuatro grupos se caracterizaron a partir de las variables de personalidad y se compararon, en función del sexo y del curso en las mismas variables. Para estas

comparaciones se empleó el test de la $X_{2}$. Además, se calcularon las razones de probabilidad (Odds Ratio) asociadas a cada comportamiento de riesgo en cada grupo respecto al resto de sujetos del mismo curso mediante el método de Newcombe. Los análisis estadísticos se realizaron con el programa SPSS vs. 15.

\section{Resultados}

Características de personalidad de la muestra y comparación entre sexos.

En la tabla 1 se presentan los datos descriptivos de la muestra en el Substance Use Risk Profile y su comparación por sexo. Se encontraron diferencias estadísticamente significativas en todas las dimensiones del SURPS entre hombres y mujeres. Los hombres presentan mayores puntuaciones en las dimensiones de búsqueda de sensaciones e impulsividad. Las mujeres presentan mayores puntuaciones en introversión y ansiedad. 
Tabla 1 : Descriptivos del Substance Use Risk Profile por sexo

\begin{tabular}{|c|c|c|c|c|c|c|c|c|}
\hline & \multicolumn{2}{|c|}{$\begin{array}{c}\text { Total } \\
(\mathrm{N}=1308)\end{array}$} & \multicolumn{2}{|c|}{$\begin{array}{l}\text { Hombre } \\
(n=675)\end{array}$} & \multicolumn{2}{|c|}{$\begin{array}{c}\text { Mujer } \\
(n=633)\end{array}$} & \multirow[t]{2}{*}{$t(g .1)}$. & \multirow[t]{2}{*}{$p$} \\
\hline & $M$ & (d.t.) & $M$ & (d.t.) & $M$ & (d.t.) & & \\
\hline Introversión & 14,61 & $(5,09)$ & 14,03 & $(4,81)$ & 15,21 & $(5,31)$ & $4,2(1279,1)$ & $<, 001$ \\
\hline $\begin{array}{l}\text { Búsqueda de } \\
\text { sensaciones }\end{array}$ & 17,88 & $(5,02)$ & 18,77 & $(5,00)$ & 16,91 & $(4,88)$ & $6,8(1319)$ & $<, 001$ \\
\hline Impulsividad & 11,09 & $(3,20)$ & 11,32 & $(3,19)$ & 10,86 & $(3,20)$ & $2,6(1305)$ &, 009 \\
\hline Ansiedad & 11,32 & $(3,79)$ & 10,68 & $(3,43)$ & 11,99 & $(4,04)$ & 6,3 (1305) & $<, 001$ \\
\hline
\end{tabular}

En la tabla 2 se presentan los percentiles 10, 50 y 90 de cada dimensión en la muestra total y en función del sexo.

Tabla 2 : Percentiles del Substance Use Risk Profile por sexo.

\begin{tabular}{llccc}
\hline & & $\begin{array}{c}\text { Total } \\
(\mathbf{N}=\mathbf{1 3 0 8})\end{array}$ & $\begin{array}{c}\text { Hombre } \\
(\mathbf{n = 6 7 5})\end{array}$ & $\begin{array}{c}\text { Mujer } \\
(\mathbf{n = 6 3 3})\end{array}$ \\
\hline \multirow{3}{*}{ Introversión } & Percentil 10 & 9,00 & 8,00 & 9,00 \\
& Percentil 50 & 14,00 & 14,00 & 15,00 \\
& Percentil 90 & 21,00 & 20,00 & 22,00 \\
\hline & & & & \\
\hline \multirow{3}{*}{ Búsqueda de sensaciones } & Percentil 10 & 11,00 & 13,00 & 10,60 \\
& Percentil 50 & 18,00 & 19,00 & 17,00 \\
& Percentil 90 & 24,00 & 25,00 & 23,00 \\
\hline & & & & \\
\hline \multirow{3}{*}{ Impulsividad } & Percentil 10 & 7,00 & 8,00 & 7,00 \\
& Percentil 50 & 11,00 & 11,00 & 10,00 \\
& Percentil 90 & 15,00 & 16,00 & 15,00 \\
\hline \multirow{3}{*}{ Ansiedad } & & & & \\
& Percentil 10 & 7,00 & 6,00 & 7,00 \\
& Percentil 50 & 11,00 & 10,00 & 11,00 \\
& Percentil 90 & 16,00 & 15,00 & 17,00 \\
\hline
\end{tabular}


Análisis de clúster y caracterización de los grupos

En la tabla 3 también se presentan los cuatro grupos obtenidos mediante el análisis de clúster y sus principales características. El grupo 1 se caracteriza por tener unas puntuaciones superiores a la media en todas las características de personalidad analizadas. El grupo 2 presenta una puntuación inferior a la media en introversión, la más alta en búsqueda de sensaciones y por encima de la media en impulsividad. El grupo 3 se caracteriza por tener la puntuación media más alta en introversión, la más baja en ansiedad y por debajo de la media en búsqueda de sensaciones e impulsividad. El grupo 4 presenta la puntuación media más baja en introversión, búsqueda de sensaciones e impulsividad y por debajo de la media en ansiedad.

Tabla 3 : Caracterización de los grupos obtenidos

\begin{tabular}{|c|c|c|c|c|c|c|c|c|c|}
\hline & \multirow{2}{*}{$\begin{array}{c}\text { Total } \\
n\end{array}$} & \multicolumn{2}{|c|}{ Grupo 1} & \multicolumn{2}{|c|}{ Grupo 2} & \multicolumn{2}{|c|}{ Grupo 3} & \multicolumn{2}{|c|}{ Grupo 4} \\
\hline & & $n$ & $\%^{1}$ & $n$ & $\%$ & $n$ & $\%$ & $n$ & $\%^{1}$ \\
\hline \multicolumn{10}{|l|}{ Personas en cada grupo } \\
\hline Total & 1308 & 246 & $18,8 \%$ & 420 & $32,1 \%$ & 274 & $21,0 \%$ & 367 & $28,1 \%$ \\
\hline Hombres & 675 & 118 & $17,5 \%$ & 271 & $40,1 \%$ & 115 & $17,0 \%$ & 171 & $25,3 \%$ \\
\hline Mujeres & 633 & 128 & $20,3 \%$ & 149 & $23,6 \%$ & 159 & $25,2 \%$ & 196 & $31,0 \%$ \\
\hline Substance Use Risk Profile & & $M$ & $P c$ & $M$ & $P c$ & $M$ & $P_{c}$ & $M$ & $P_{c}$ \\
\hline Introversión & & 18,83 & 80 & 11,79 & 30 & 19,92 & 85 & 11,57 & 30 \\
\hline Búsqueda sensaciones & & $\begin{array}{c}20,5 \\
3\end{array}$ & 70 & $\begin{array}{c}22,2 \\
2\end{array}$ & 85 & 14,48 & 20 & 14,34 & 20 \\
\hline Impulsividad & & 13,11 & 75 & 11,82 & 70 & 10,65 & 55 & 9,24 & 30 \\
\hline Ansiedad & & 14,57 & 85 & 10,36 & 45 & 10,09 & 45 & 11,16 & 55 \\
\hline
\end{tabular}

$1 \%$ sobre total fila; $P C=$ Percentil de la media

Conductas de riesgo entre los hombres en función del grupo

En la tabla 4 se presentan las conductas de riesgo que se han realizado alguna vez en la vida y la repetición de curso entre los hombres. El $67,8 \%$ de los hombres ha tomado alcohol, el $36,6 \%$ ha fumado tabaco, el $32,1 \%$ ha fumado cánnabis y el $10,7 \%$ ha tenido relaciones sexuales sin protección. En cuanto a la evolución en los comportamientos, en $2^{\circ}$ de la ESO la prevalencia de consumo de alcohol es del $38,1 \%$, para pasar al $84,6 \%$ en $4^{\circ}$ de la ESO. Una evolución semejante se da en el consumo de tabaco (del $13,83 \%$ al $50,6 \%$ ) y en el consumo de cánnabis (del 11,2 al 40,1\%). En lo que respecta a las relaciones sexuales sin protección, el mayor incremento se da entre $4^{\circ}$ de la ESO y los mayores de 16 años (del 8,7\% al 20,4\%). Un $31,1 \%$ ha repetido curso, alcanzado un $51,3 \%$ en los mayores de 16 años. 
Tabla 4 : Conductas de riesgo (alguna vez en la vida) en función del grupo (hombres)

\begin{tabular}{|c|c|c|c|c|c|c|c|c|c|c|c|c|c|c|c|c|}
\hline & \multicolumn{2}{|c|}{$\begin{array}{c}\text { Total } \\
(\mathrm{N}=666)\end{array}$} & \multicolumn{3}{|c|}{$\begin{array}{l}\text { Grupo } 1 \\
(n=118)\end{array}$} & \multicolumn{3}{|c|}{$\begin{array}{l}\text { Grupo } 2 \\
(n=268)\end{array}$} & \multicolumn{3}{|c|}{$\begin{array}{c}\text { Grupo } 3 \\
(n=112)\end{array}$} & \multicolumn{3}{|c|}{$\begin{array}{l}\text { Grupo } 4 \\
(n=169)\end{array}$} & \multirow{2}{*}{$\begin{array}{c}X^{2} \\
g . l .=3\end{array}$} & \multirow{2}{*}{$p$} \\
\hline & n & $\%{ }^{1}$ & $\mathbf{n}$ & $\%^{2}$ & OR & n & $\%^{2}$ & OR & $\mathbf{n}$ & $\%^{2}$ & OR & $\mathbf{n}$ & $\%^{2}$ & OR & & \\
\hline \multicolumn{17}{|c|}{ Han fumado cigarrillos } \\
\hline Total $(N=666)$ & 244 & $36,60 \%$ & 62 & $52,50 \%$ & 2,23 & 103 & $38,43 \%$ & 1,14 & 46 & $41,07 \%$ & 1,26 & 33 & $19,53 \%$ & 0,33 & 35,5 & 000 \\
\hline $2 \mathrm{ESO}(\mathrm{N}=253)$ & 35 & $13,83 \%$ & 10 & $8,47 \%$ & 2,61 & 14 & $5,22 \%$ & 1,02 & 6 & $5,36 \%$ & 1,40 & 5 & $2,96 \%$ & 0,32 & 8,8 & 032 \\
\hline $4 \mathrm{ESO}(\mathrm{N}=229)$ & 116 & $50,61 \%$ & 28 & $23,73 \%$ & 2,25 & 56 & $20,90 \%$ & 1,70 & 23 & $20,54 \%$ & 1,15 & 9 & $5,33 \%$ & 0,16 & 26,2 &, 000 \\
\hline$>16$ años $(N=185)$ & 93 & $50,23 \%$ & 24 & $20,34 \%$ & 2,11 & 33 & $12,31 \%$ & 0,75 & 17 & $15,18 \%$ & 0,92 & 19 & $11,24 \%$ & 0,82 & 4,0 & 260 \\
\hline \multicolumn{17}{|c|}{ Han tomado alcohol } \\
\hline Total $(N=666)$ & 452 & $67,82 \%$ & 92 & $77,97 \%$ & 1,85 & 202 & $75,37 \%$ & 1,81 & 71 & $63,39 \%$ & 0,81 & 87 & $51,48 \%$ & 0,38 & 34,0 &, 000 \\
\hline $2 \mathrm{ESO}(\mathrm{N}=254)$ & 97 & $38,14 \%$ & 21 & $17,80 \%$ & 2,13 & 52 & $19,40 \%$ & 2,62 & 8 & $7,14 \%$ & 0,43 & 16 & $9,47 \%$ & 0,29 & 26,8 &, 000 \\
\hline $4 \mathrm{ESO}(\mathrm{N}=228)$ & 193 & $84,61 \%$ & 38 & $32,20 \%$ & 1,90 & 86 & $32,09 \%$ & 2,01 & 33 & $29,46 \%$ & 0,60 & 36 & $21,30 \%$ & 0,44 & 7,5 &, 057 \\
\hline$>16$ años $(N=184)$ & 162 & $88,01 \%$ & 33 & $27,97 \%$ & 1,15 & 64 & $23,88 \%$ & 1,14 & 30 & $26,79 \%$ & 1,02 & 35 & $20,71 \%$ & 0,73 & 0,37 & 945 \\
\hline \multicolumn{17}{|c|}{ Han fumado cánnabis } \\
\hline Total $(N=663)$ & 213 & $32,13 \%$ & 56 & $47,46 \%$ & 2,23 & 90 & $33,58 \%$ & 1,15 & 39 & $34,82 \%$ & 1,16 & 28 & $16,57 \%$ & 0,34 & 31,9 &, 000 \\
\hline $2 \mathrm{ESO}(\mathrm{N}=249)$ & 28 & $11,21 \%$ & 10 & $8,47 \%$ & 3,68 & 11 & $4,10 \%$ & 1,02 & 5 & $4,46 \%$ & 1,44 & 2 & $1,18 \%$ & 0,14 & 14,5 &, 002 \\
\hline $4 \mathrm{ESO}(\mathrm{N}=229)$ & 92 & $40,12 \%$ & 23 & $19,49 \%$ & 2,07 & 43 & $16,04 \%$ & 1,39 & 17 & $15,18 \%$ & 0,97 & 9 & $5,33 \%$ & 0,27 & 13,7 &, 003 \\
\hline$>16$ años $(N=185)$ & 93 & $50,21 \%$ & 23 & $19,49 \%$ & 1,83 & 36 & $13,43 \%$ & 0,98 & 17 & $15,18 \%$ & 0,92 & 17 & $10,06 \%$ & 0,63 & 3,4 &, 333 \\
\hline \multicolumn{17}{|c|}{ Relación sexual sin protección } \\
\hline Total $(N=672)$ & 72 & $10,71 \%$ & 23 & $19,49 \%$ & 2,52 & 31 & $11,57 \%$ & 1,13 & 12 & $10,71 \%$ & 0,98 & 6 & $3,55 \%$ & 0,24 & 19,1 & 000 \\
\hline $2 \mathrm{ESO}(\mathrm{N}=256)$ & 14 & $5,52 \%$ & 7 & $5,93 \%$ & 6,81 & 4 & $1,49 \%$ & 0,59 & 2 & $1,79 \%$ & 1,06 & 1 & $0,59 \%$ & 0,16 & 15,6 & ,001 \\
\hline $4 \mathrm{ESO}(\mathrm{N}=230)$ & 20 & $8,73 \%$ & 6 & $5,08 \%$ & 2,07 & 11 & $4,10 \%$ & 1,76 & 2 & $1,79 \%$ & 0,46 & 1 & $0,59 \%$ & 0,18 & 6,0 &, 110 \\
\hline$>16$ años $(N=186)$ & 38 & $20,42 \%$ & 10 & $8,47 \%$ & 1,60 & 16 & $5,97 \%$ & 1,19 & 8 & $7,14 \%$ & 1,14 & 4 & $2,37 \%$ & 0,35 & 4,0 & 253 \\
\hline \multicolumn{17}{|c|}{ Han repetido algún curso } \\
\hline Total $(N=673)$ & 209 & $31,12 \%$ & 53 & $44,92 \%$ & 2,09 & 73 & $27,24 \%$ & 0,72 & 42 & $37,50 \%$ & 1,35 & 41 & $24,26 \%$ & 0,64 & 18,0 &, 000 \\
\hline $2 \mathrm{ESO}(\mathrm{N}=257)$ & 53 & $20,62 \%$ & 13 & $11,02 \%$ & 2,23 & 20 & $7,46 \%$ & 0,90 & 9 & $8,04 \%$ & 1,40 & 11 & $6,51 \%$ & 0,50 & 7,0 & 074 \\
\hline $4 \mathrm{ESO}(\mathrm{N}=229)$ & 60 & $26,23 \%$ & 14 & $11,86 \%$ & 1,53 & 22 & $8,21 \%$ & 0,73 & 14 & $12,50 \%$ & 1,47 & 10 & $5,92 \%$ & 0,71 & 3,2 & 360 \\
\hline$>16$ años $(N=187)$ & 96 & $51,34 \%$ & 26 & $22,03 \%$ & 2,70 & 31 & $11,57 \%$ & 0,58 & 19 & $16,96 \%$ & 1,00 & 20 & $11,83 \%$ & 0,88 & 7,4 & 060 \\
\hline
\end{tabular}

\section{Conductas de riesgo entre las mujeres en función del grupo}

En la tabla 5 se presentan las conductas de riesgo (realizadas alguna vez en la vida) y la repetición de curso entre las mujeres.

Tabla 5: Conductas de riesgo (alguna vez en la vida) en función del grupo (mujeres)

\begin{tabular}{|c|c|c|c|c|c|c|c|c|c|c|c|c|c|c|c|c|}
\hline & \multicolumn{2}{|c|}{$\begin{array}{c}\text { Total } \\
(\mathrm{N}=632)\end{array}$} & \multicolumn{3}{|c|}{$\begin{array}{l}\text { Grupo } 1 \\
(n=128)\end{array}$} & \multicolumn{3}{|c|}{$\begin{array}{l}\text { Grupo 2 } \\
(n=149)\end{array}$} & \multicolumn{3}{|c|}{$\begin{array}{l}\text { Grupo } 3 \\
(n=159)\end{array}$} & \multicolumn{3}{|c|}{$\begin{array}{r}\text { Grupo } 4 \\
(n=196)\end{array}$} & \multirow{2}{*}{$\begin{array}{c}x^{2} \\
g . l .=3\end{array}$} & \multirow[t]{2}{*}{$p$} \\
\hline & $\mathbf{n}$ & $\%$ & $\mathbf{n}$ & $\%^{2}$ & OR & $\mathbf{n}$ & $\%^{2}$ & OR & $\mathbf{n}$ & $\%^{2}$ & OR & $\mathbf{n}$ & $\%^{2}$ & OR & & \\
\hline \multicolumn{17}{|c|}{ Han fumado cigarrillos } \\
\hline Total $(N=630)$ & 252 & $40,02 \%$ & 76 & $59,38 \%$ & 2,71 & 72 & $48,32 \%$ & 1,62 & 65 & $40,88 \%$ & 1,05 & 39 & $19,90 \%$ & 0,26 & 58,0 &, 000 \\
\hline $2 \mathrm{ESO}(\mathrm{N}=205)$ & 21 & $10,21 \%$ & 6 & $4,69 \%$ & 2,67 & 7 & $4,70 \%$ & 1,59 & 6 & $3,77 \%$ & 2,05 & 2 & $1,02 \%$ & 0,12 & 11,4 &, 009 \\
\hline $4 \mathrm{ESO}(\mathrm{N}=249)$ & 123 & $49,43 \%$ & 40 & $31,25 \%$ & 2,71 & 30 & $20,13 \%$ & 1,61 & 37 & $23,27 \%$ & 1,12 & 16 & $8,16 \%$ & 0,22 & 27,3 &, 000 \\
\hline$>16$ años $(N=176)$ & 108 & $61,42 \%$ & 30 & $23,44 \%$ & 2,52 & 35 & $23,49 \%$ & 2,78 & 22 & $13,84 \%$ & 0,34 & 21 & $10,71 \%$ & 0,58 & 18,0 &, 000 \\
\hline \multicolumn{17}{|l|}{ Han tomado alcohol } \\
\hline Total $(\mathrm{N}=631)$ & 413 & $65,51 \%$ & 102 & $79,69 \%$ & 2,42 & 107 & $71,81 \%$ & 1,51 & 111 & $69,81 \%$ & 1,30 & 93 & $47,45 \%$ & 0,32 & 43,9 &, 000 \\
\hline $2 \mathrm{ESO}(\mathrm{N}=205)$ & 49 & $23,92 \%$ & 12 & $9,38 \%$ & 2,49 & 17 & $11,41 \%$ & 1,91 & 13 & $8,18 \%$ & 2,09 & 7 & $3,57 \%$ & 0,15 & 22,0 &, 000 \\
\hline $4 \mathrm{ESO}(\mathrm{N}=249)$ & 204 & $81,94 \%$ & 52 & $40,63 \%$ & 1,86 & 45 & $30,20 \%$ & 1,84 & 59 & $37,11 \%$ & 1,00 & 48 & $24,49 \%$ & 0,42 & 7,7 &, 053 \\
\hline$>16$ años $(N=177)$ & 160 & $90,43 \%$ & 38 & $29,69 \%$ & 4,98 & 45 & $30,20 \%$ & 6,26 & 39 & $24,53 \%$ & 0,13 & 38 & $19,39 \%$ & 1,45 & 16,8 &, 001 \\
\hline \multicolumn{17}{|c|}{ Han fumado cánnabis } \\
\hline Total $(N=630)$ & 157 & $24,92 \%$ & 53 & $41,41 \%$ & 2,75 & 48 & $32,21 \%$ & 1,64 & 36 & $22,64 \%$ & 0,85 & 20 & $10,20 \%$ & 0,25 & 46,7 &, 000 \\
\hline $2 \mathrm{ESO}(\mathrm{N}=205)$ & 12 & $5,92 \%$ & 4 & $3,13 \%$ & 3,21 & 5 & $3,36 \%$ & 2,28 & 3 & $1,89 \%$ & 1,62 & 0 & - & 0 & 10,4 &, 016 \\
\hline 4 ESO $(N=249)$ & 67 & $26,91 \%$ & 26 & $20,31 \%$ & 2,86 & 16 & $10,74 \%$ & 1,32 & 18 & $11,32 \%$ & 0,87 & 7 & $3,57 \%$ & 0,24 & 18,7 &, 000 \\
\hline$>16$ años $(N=176)$ & 78 & $44,31 \%$ & 23 & $17,97 \%$ & 2,31 & 27 & $18,12 \%$ & 2,20 & 15 & $9,43 \%$ & 0,41 & 13 & $6,63 \%$ & 0,50 & 15,1 &, 002 \\
\hline \multicolumn{17}{|c|}{ Relación sexual sin protección } \\
\hline Total $(N=628)$ & 64 & $10,23 \%$ & 19 & $14,84 \%$ & 1,78 & 23 & $15,44 \%$ & 1,97 & 13 & $8,18 \%$ & 0,74 & 9 & $4,59 \%$ & 0,33 & 15,1 &, 002 \\
\hline $2 \mathrm{ESO}(\mathrm{N}=203)$ & 1 & $0,54 \%$ & 0 & - & -- & 1 & $0,67 \%$ & - & 0 & $40,25 \%$ & -- & 0 & - & -- & 3,0 & 380 \\
\hline $4 \mathrm{ESO}(\mathrm{N}=248)$ & 19 & $7,71 \%$ & 7 & $5,47 \%$ & 2,04 & 5 & $3,36 \%$ & 1,42 & 5 & $3,14 \%$ & 0,86 & 2 & $1,02 \%$ & 0,30 & 4,0 & 256 \\
\hline$>16$ años $(N=177)$ & 44 & $24,92 \%$ & 12 & $9,38 \%$ & 1,47 & 17 & $11,41 \%$ & 2,16 & 8 & $5,03 \%$ & 0,48 & 7 & $3,57 \%$ & 0,55 & 7,4 &, 061 \\
\hline \multicolumn{17}{|c|}{ Han repetido algún curso } \\
\hline Total $(N=627)$ & 150 & $23,89 \%$ & 40 & $31,25 \%$ & 1,63 & 23 & $15,44 \%$ & 0,51 & 57 & $35,85 \%$ & 2,31 & 30 & $15,31 \%$ & 0,47 & 30,7 &, 000 \\
\hline $2 \mathrm{ESO}(\mathrm{N}=203)$ & 27 & $13,34 \%$ & 6 & $4,69 \%$ & 1,81 & 5 & $3,36 \%$ & 0,39 & 11 & $6,92 \%$ & 4,35 & 5 & $2,55 \%$ & 0,25 & 16,0 &, 001 \\
\hline $4 \mathrm{ESO}(\mathrm{N}=248)$ & 59 & $23,81 \%$ & 19 & $14,84 \%$ & 1,83 & 7 & $4,70 \%$ & 0,44 & 19 & $11,95 \%$ & 1,22 & 14 & $7,14 \%$ & 0,80 & 6,0 &, 112 \\
\hline$>16$ años $(N=176)$ & 64 & $36,43 \%$ & 15 & $11,72 \%$ & 1,12 & 11 & $7,38 \%$ & 0,44 & 27 & $16,98 \%$ & 2,82 & 11 & $5,61 \%$ & 0,59 & 11,5 &, 009 \\
\hline
\end{tabular}

$1 \%$ sobre total fila; ${ }^{2} \%$ sobre total columna; $O R=$ Odds Ratio de cada grupo respecto al resto de sujetos de la fila 
El $40 \%$ de las mujeres han fumado tabaco, el $65,5 \%$ ha tomado alcohol, el $24,9 \%$ ha fumado cánnabis, el $10,2 \%$ ha tenido relaciones sexuales sin protección y el $23,9 \%$ ha repetido algún curso. En términos de probabilidad, las mujeres del grupo 1 presentan unas mayores Odds Ratio en consumo de tabaco, cánnabis y alcohol. Las mujeres del grupo 2 presentan la mayor Odds Ratio de relaciones sexuales sin protección y las del grupo 3 de repetir algún curso académico.

\section{Discusión}

Los objetivos del presente trabajo eran establecer la prevalencia de diferentes comportamientos de riesgo en una muestra de adolescentes, determinar las cuatro dimensiones de personalidad en dicha muestra, y establecer perfiles de personalidad en función de los comportamientos de riesgo. El principal hallazgo de este estudio ha sido que los perfiles de personalidad encontrados discriminan mejor las conductas de riesgo y la repetición de curso que la comparación a partir de las dimensiones de personalidad por separado. Este resultado es divergente al encontrado por Malmberg (Malmberg et al., 2012). Una de las causas de esta diferencia puede ser el análisis de datos realizado. En este estudio se ha realizado un análisis de clúster. Se trata de una prueba inductiva que clasifica a un conjunto de individuos en grupos homogéneos a partir de una visión conjunta de las dimensiones de personalidad, en lugar de considerar cada una por separado. Éste es un enfoque más ecológico que el de las clases latentes (usado por Malmberg), basado en la agrupación de las variables, no de los individuos. Además, en este estudio la identificación del sexo se ha hecho después de haber realizado el análisis de clúster, por lo que el resultado encontrado puede ser más fiel a lo que ocurre en la realidad.

Cuando se consideran los datos de la muestra en su conjunto se observa, en líneas generales, que la prevalencia de comportamientos de riesgo encontrados es semejante a la de otras muestras españolas. En cuanto al consumo de sustancias, la principal sustancia con la que han experimentado los adolescentes es el alcohol, tal y como recogen los datos de otros estudios (Observatorio Español de las Drogas y las Adicciones, 2020). Éste es un dato preocupante, puesto que todos los participantes en este estudio son menores de edad, y a pesar de no estar permitido el suministro de alcohol a menores de 18 años, dos de cada tres ya lo han probado, sin encontrar diferencias en el consumo entre hombres y mujeres. El tabaco (sin diferencias entre hombres y mujeres) y en una proporción menor el cánnabis (con un mayor porcentaje de hombres) han sido experimentadas por alrededor del $40 \%$ de los menores. Todos estos datos destacan la importancia de considerar el consumo de sustancias en los adolescentes y la de revisar los programas de prevención que se hayan podido aplicar. 
Por lo que se refiere a las relaciones sexuales sin protección, uno de cada diez adolescentes ha realizado este tipo de prácticas sin encontrarse diferencias entre hombres y mujeres. Este dato es semejante al de un estudio realizado también en Navarra y remarca la necesidad de seguir interviniendo sobre este tipo de riesgos en la adolescencia (González-Eransus \& Miqueléiz, 2016).

En lo concerniente al rendimiento escolar, los datos también son semejantes a los encontrados en otros estudios (Ministerio de Educación y Formación Profesional, 2019). Casi uno de cada tres hombres ha repetido algún curso, mientras que entre las mujeres esta proporción se reduce a una de cada cuatro.

El tránsito de $2^{\circ}$ a $4^{\circ}$ de la Educación Secundaria Obligatoria (E.S.O.) es el que presenta, en líneas generales, un mayor incremento en la realización de las diferentes conductas de riesgo. Así pues, parece que las intervenciones preventivas deberían realizarse antes de $4^{\circ}$ de la E.S.O.

En cierto sentido, la principal aportación de este artículo radica en la categorización de los adolescentes en cuatro grupos según sus características de personalidad. A partir de la clasificación obtenida, se han encontrado diferencias estadísticamente significativas en la mayoría de los comportamientos analizados tanto entre los diferentes cursos escolares como en la muestra en su conjunto, validando las agrupaciones establecidas por el análisis de clúster.

El grupo 1 es el de mayor riesgo para el consumo de drogas tanto en hombres como en mujeres, y además los hombres de este grupo también presentan una mayor probabilidad de mantener relaciones sexuales sin protección y de repetir curso. Por el contrario, el grupo 4 (tanto en hombres como en mujeres) es el que presenta una menor probabilidad de realizar comportamientos de riesgo. Las mujeres del grupo 3 son las que tienen una mayor probabilidad de repetir curso, y las mujeres del grupo 2 de mantener relaciones sexuales sin protección.

Los adolescentes del grupo 1 se caracterizan por tener unas puntuaciones superiores a la media en todas las características de personalidad analizadas. La alta presencia de comportamientos de riesgo se explicaría por las altas puntuaciones en búsqueda de sensaciones e impulsividad y por la vinculación entre repetición escolar y comportamientos de riesgo. La sensibilidad a la ansiedad ha mostrado su asociación con altos niveles de consumo de alcohol y de otras drogas (Conrod et al., 2000). También se ha encontrado un inicio 
temprano del consumo de alcohol asociado a la desesperanza (Conrod et al., 2010). Y tanto la sensibilidad a la ansiedad como la desesperanza se han vinculado en diferentes investigaciones, al consumo de sustancias y otros comportamientos antisociales de alto riesgo (Conrod, Castellanos, \& Mackie, 2008; Krueger et al., 2002). Por lo tanto, los sujetos pertenecientes al grupo 1 deberían recibir una atención especial ya que destacan como una población con muy alto riesgo. Además, entre los chicos mayores de 16 años, es el grupo que mayor porcentaje presenta de repetidores. Con este alumnado se recomienda una prevención indicada e individualizada dado el alto riesgo que presentan (Edalati et al., 2019).

El grupo 4 que presenta menores tasas de comportamiento de riesgo, está caracterizado por bajos niveles de introversión, de búsqueda de sensaciones y de impulsividad. Este dato es coherente con los resultados obtenidos en estudios previos que vinculan altos niveles de búsqueda de sensaciones e impulsividad con mayores consumos de sustancias y más conductas de riesgo. Estos adolescentes coincidirían con el grupo de los "resilientes" de Malmberg et al. (2012).

Este grupo fue categorizado como "internalizador" por Malmberg. En línea con lo esperado se ha asociado con un porcentaje menor de experimentación en alcohol, tabaco, cánnabis o relaciones sexuales sin protección que los grupos 1 y 2 . Un resultado no esperado en este grupo es que las mujeres presentan un mayor porcentaje de repeticiones de curso. La alta introversión podría ser un reflejo del mayor número de repeticiones de curso encontrado en este grupo lo cual incidiría en una menor autoestima académica (Sánchez-Sosa, VillarrealGonzález, Ávila Guerrero, Vera Jiménez, \& Musitu, 2014). También podría apuntar a una peor socialización, que les llevaría a aislarse del resto de comportamientos seguidos por sus compañeros, ya que se ha encontrado entre las mujeres una tendencia mayor a aislar a las compañeras que no comparten los intereses del grupo que entre los chicos (Peñalva-Vélez, López-Goñi, \& García-Manso, 2016). En cualquier caso, las actividades preventivas con las mujeres de este grupo 3 deberían contemplar una atención específica al desempeño académico.

El grupo 3 se caracteriza por tener una mayor introversión, y una menor búsqueda de sensaciones e impulsividad. El grupo 2, con baja introversión y alta búsqueda de sensaciones e impulsividad es semejante al grupo "externalizante" de Malmberg, y es el opuesto al grupo 3. A diferencia del grupo 3, es el que mayor porcentaje de relaciones sexuales sin protección presenta entre las mujeres. Así pues, en este caso las actividades preventivas deberían contemplar intervenciones sobre las conductas sexuales de riesgo entre las mujeres de este grupo. 
La principal limitación de este estudio es que se trata de un estudio basado en un cuestionario autoinformado, que podría tener errores de medida debidas a causas situacionales y cognitivas (Brener, Billy, \& Grady, 2003). En este sentido, y con el propósito de minimizar estos sesgos el equipo investigador aseguró a los participantes la confidencialidad de las respuestas. Por otro lado, este artículo se ha centrado en el inicio del consumo de sustancias, dejando para posteriores investigaciones la frecuencia y los patrones de consumo de estos adolescentes. A partir de los datos presentados en esta investigación se puede profundizar en las características de los patrones de consumo y las características de personalidad asociadas. Finalmente, hay que tener en cuenta que la muestra ha sido recogida en la Comunidad de Navarra, comunidad que cuenta con buenos servicios sociales y que puede diferir de otras zonas con menores niveles de protección social (Rueda, 2012).

En conclusión, a partir de las dimensiones de personalidad del SURPS se han podido establecer cuatro diferentes perfiles de adolescentes en relación a las conductas de riesgo analizadas, destacándose las diferencias entre hombres y mujeres en relación al desempeño académico y señalándose las estrategias preventivas que deberían proponerse en cada subgrupo. Finalmente, sería deseable poder realizar estudios prospectivos que valoren la evolución de los adolescentes a partir de las dimensiones de personalidad aquí evaluadas, tal y como se ha realizado en otros países (Edalati \& Conrod, 2019).

\section{Reconocimientos}

Los autores desean agradecer al profesorado implicado las facilidades que han dado para la realización de la presente investigación.

Los autores informan de que no tienen conflictos de interés ni han recibido financiación alguna para la elaboración de este artículo.

\section{Referencias}

Adan, A. (2012). Impulsividad funcional y disfuncional en jóvenes con consumo intensivo de alcohol (binge drinking). Adicciones, 24(1), 17-22. https://doi.org/10.20882/adicciones.113

Aleixandre, N. L., Pol, A. P., \& del Río, M. J. P. (2005). Características de personalidad en adolescentes como predictores de la conducta de consumo de sustancias psicoactivas. Trastornos Adictivos, 7(2), 90-96. https://doi.org/10.1016/S1575-0973(05)74513-X 
Arnett, J. (1994). Sensation seeking: A new conceptualization and a new scale. Personality and Individual Differences, 16(2), 289-296. https://doi.org/10.1016/0191-8869(94)90165-1

Arthur, M. W., Hawkins, J. D., Pollard, J. A., Catalano, R. F., \& Baglioni, A. J. J. (2002). Measuring risk and protective factors for use, delinquency, and other adolescent problem behaviors the communities that care youth survey. Evaluation Review, 26(6), 575-601. https://doi.org/10.1177/0193841X0202600601

Beck, K. H., Caldeira, K. M., Vincent, K. B., O'Grady, K. E., Wish, E. D., \& Arria, A. M. (2009). The social context of cannabis use: relationship to cannabis use disorders and depressive symptoms among college students. Addictive Behaviors, 34(9), 764-768. https://doi.org/10.1016/j.addbeh.2009.05.001

Brener, N. D., Billy, J. O. G., \& Grady, W. R. (2003). Assessment of factors affecting the validity of self-reported health-risk behavior among adolescents: evidence from the scientific literature. Journal of Adolescent Health, 33(6), 436-457. https://doi.org/10.1016/S1054$139 \times(03) 00052-1$

Cano-Vindel, A., Miguel-Tobal, J. J., González, H., \& Iruarrizaga, I. (1994). El afrontamiento de la ansiedad en las drogodependencias. Anales de Psicología, 10(2), 145-156. https://tinyurl.com/yy6jkxse

Carceller-Maicas, N., Ariste, S., Martínez-Hernáez, A., Martorell-Poveda, M. A., Correa-Urquiza, M., \& DiGiacomo, S. M. (2015). El consumo de tabaco como automedicación de depresión/ansiedad entre los jóvenes: resultados de un estudio con método mixto. Adicciones, 26(1), 34-45. https://doi.org/10.20882/adicciones.127

Castellanos, N., \& Conrod, P. (2006). Brief interventions targeting personality risk factors for adolescent substance misuse reduce depression, panic and risk-taking behaviours. Journal of Mental Health, 15(6), 645-658. https://doi.org/10.1080/09638230600998912

Castellanos-Ryan, N., Rubia, K., \& Conrod, P. J. (2011). Response inhibition and reward response bias mediate the predictive relationships between impulsivity and sensation seeking and common and unique variance in conduct disorder and substance misuse. Alcoholism: Clinical and Experimental Research, 35(1), 140-155. https://doi.org/10.1111/j.15300277.2010.01331.x

Conrod, P. J., Castellanos, N., \& Mackie, C. (2008). Personality-targeted interventions delay the growth of adolescent drinking and binge drinking. Journal of Child Psychology and Psychiatry, 49(2), 181-190. https://doi.org/10.1111/j.1469-7610.2007.01826.x 
Conrod, P. J., Castellanos-Ryan, N., \& Strang, J. (2010). Brief, personality-targeted coping skills interventions and survival as a non-drug user over a 2-year period during adolescence. Archives of General Psychiatry, 67(1), 85-93. https://doi.org/10.1001/ archgenpsychiatry.2009.173

Conrod, P. J., Pihl, R. O., Stewart, S. H., \& Dongier, M. (2000). Validation of a system of classifying female substance abusers on the basis of personality and motivational risk factors for substance abuse. Psychology of Addictive Behaviors, 14(3), 243-256. https://doi.org/ $10.1037 / / 0893-164 x .14 .3 .243$

Croese, E. M., \& van Hoof, J. J. (2020). Alcohol Intoxication and Psychological Problems Among Adolescents. Journal of Child \& Adolescent Substance Abuse, 28(6), 462-473. https://doi.org/10.1080/1067828X.2020.1789524

Edalati, H., Afzali, M. H., Castellanos-Ryan, N., \& Conrod, P. J. (2019). The Effect of Contextual Risk Factors on the Effectiveness of Brief Personality-Targeted Interventions for Adolescent Alcohol Use and Misuse: A Cluster-Randomized Trial. Alcoholism-Clinical and Experimental Research, 43(5), 997-1006. https://doi.org/10.1111/acer.14016

Edalati, H., \& Conrod, P. J. (2019). A Review of Personality-Targeted Interventions for Prevention of Substance Misuse and Related Harm in Community Samples of Adolescents. Frontiers in Psychiatry, 9, Art. ID: 770. https://doi.org/10.3389/fpsyt.2018.00770

Farías, M., Fiol, D., Kit, I., \& Melgar, S. (2007). Todos Pueden Aprender. Propuestas para superar el fracaso escolar. Buenos Aires: Fondo de las Naciones Unidas para la infancia y Asociación civil educación para todos. https://tinyurl.com/y4ugsdbz

Fernie, G., Peeters, M., Gullo, M. J., Christiansen, P., Cole, J. C., Sumnall, H., \& Field, M. (2013). Multiple behavioural impulsivity tasks predict prospective alcohol involvement in adolescents. Addiction, 108(11), 1916-1923. https://doi.org/10.1111/add.12283

Glaser, R. R., Horn, M. L. V, Arthur, M. W., Hawkins, J. D., \& Catalano, R. F. (2005). Measurement properties of the Communities That Care ${ }^{\circledR}$ Youth Survey across demographic groups. Journal of Quantitative Criminology, 21(1), 73-102. https://doi.org/10.1007/s10940004-1788-1

González-Eransus, R., \& Miqueléiz, E. (2016). La salud de la juventud navarra. Encuesta sobre Juventud y Salud. Pamplona: Instituto de Salud Pública y Laboral de Navarra. https://tinyurl.com/y3dbrb2l 
Gonzálvez, M. T., Espada, J. P., \& Orgilés, M. (2015). Estado de ánimo y consumo de tabaco en una muestra de adolescentes españoles. Revista Latinoamericana de Psicología, 47(2), 86-92. https://doi.org/10.1016/j.rlp.2015.05.001

Jurk, S., Kuitunen-Paul, S., Kroemer, N. B., Artiges, E., Banaschewski, T., Bokde, A. L. W., . . Flor, H. (2015). Personality and substance use: psychometric evaluation and validation of the Substance Use Risk Profile Scale (SURPS) in English, Irish, French, and German adolescents. Alcoholism: Clinical and Experimental Research, 39(11), 2234-2248. https://doi.org/10.1111/acer.12886

Krank, M., Stewart, S. H., O'Connor, R., Woicik, P. B., Wall, A. M., \& Conrod, P. J. (2011). Structural, concurrent, and predictive validity of the Substance Use Risk Profile Scale in early adolescence. Addictive Behaviors, 36(1-2), 37-46. https://doi.org/10.1016/j.addbeh.2 010.08.010

Krueger, R. F., Hicks, B. M., Patrick, C. J., Carlson, S. R., lacono, W. G., \& McGue, M. (2002). Etiologic connections among substance dependence, antisocial behavior, and personality: modeling the externalizing spectrum. Journal of Abnormal Psychology, 111(3), 411-424. https://doi.org/10.1037/0021-843X.111.3.411

Lammers, J., Kuntsche, E., Engels, R. C., Wiers, R. W., \& Kleinjan, M. (2013). Mediational relations of substance use risk profiles, alcohol-related outcomes, and drinking motives among young adolescents in the Netherlands. Drug and Alcohol Dependence, 133(2), 571579.https://doi.org/10.1016/j.drugalcdep.2013.07.030

Leeman, R. F., Hoff, R. A., Krishnan-Sarin, S., Patock-Peckham, J. A., \& Potenza, M. N. (2014). Impulsivity, sensation-seeking, and part-time job status in relation to substance use and gambling in adolescents. Journal of Adolescent Health, 54(4), 460-466. https://doi.org/10.1016/j.jadohealth.2013.09.014

López-Larrosa, S. \& Rodríguez-Arias, J. L. (2012). Factores de riesgo y de protección en el consumo de drogas y la conducta antisocial en adolescentes y jóvenes españoles. International Journal of Psychological Research, 5(1), 25-33. https://tinyurl.com/y6gu359j

Lucchese, S.M., Burrone, M. S., Enders, J. E., Fernández, A. R. (2013). Consumo de sustancias y escuela: un estudio en adolescentes escolarizados de Córdoba. Revista de Salud Pública, 17(1), 32-41. https://doi.org/10.31052/1853.1180.v17.n1.6821 
Malmberg, M., Kleinjan, M., Vermulst, A. A., Overbeek, G., Monshouwer, K., Lammers, J., \& Engels, R. C. M. E. (2012). Do substance use risk personality dimensions predict the onset of substance use in early adolescence? A variable-and person-centered approach. Journal of Youth and Adolescence, 41(11), 1512-1525. https://doi.org/10.1007/s10964-0129775-6

Martín, A. B. (2007). La LOE ante el fracaso, la repetición y el abandono escolar. Revista lberoamericana de educación, 43(7), 1-11. https://tinyurl.com/yyeng8xq

Ministerio de Educación y Formación Profesional (2019). PISA 2018. Programa para la Evaluación Internacional de los Estudiantes. Informe español. Madrid: Autor. https://tinyurl.com /ycvrcwpz

Newton, N. C., Barrett, E. L., Castellanos-Ryan, N., Kelly, E., Champion, K. E., Stapinski, L., . . Teesson, M. (2016). The validity of the Substance Use Risk Profile Scale (SURPS) among Australian adolescents. Addictive Behaviors, 53, 23-30. https://doi.org/10.1016/ j.addbeh.2015.09.015

Observatorio de Salud Sexual y Reproductiva de la Sociedad Española de Contracepción (2019). Estudio sobre sexualidad y anticoncepción: jóvenes españoles. Madrid: Sociedad Española de Contracepción. https://tinyurl.com/yy8tccag

Observatorio Español de las Drogas y las Adicciones (2020). Encuesta sobre uso de drogas en enseñanzas secundarias en España (ESTUDES), 1994-2018/2019. En: Informe 2020 Alcohol, tabaco y drogas ilegales en España. Madrid: Ministerio de Sanidad. https://tinyurl.com/y53hhre5

Peñalva-Vélez, A., López-Goñi, J.-J., \& García-Manso, M.-I. (2016). El desarrollo de las competencias emocionales en alumnado de secundaria: perfiles diferenciales en función del sexo. Educatio Siglo XXI, 34(1), 223-242. https://doi.org/10.6018/j/253301

Pérez de Albéniz-Garrote, G. \& Medina, B. (2019). Influencia de la impulsividad y de la búsqueda de sensaciones en el consumo precoz de cannabis. Diferencias de género y orientaciones para la prevención. Revista de Orientación y Psicopedagogía, 30(1), 27-40. https://doi.org/10.5944/reop.vol.30.num.1.2019.25192

Pérez-Fuentes, M., Gázquez, J. J., del Mar Molero, M., Cardila, F., Martos, Á., Barragán, A. B., . . Mercader, I. (2015). Impulsividad y consumo de alcohol y tabaco en adolescentes. European Journal of Investigation in Health, Psychology and Education, 5(3), 371-382. https://doi.org/10.30552/ejihpe.v5i3.139

Pinazo Hernandis, S., Diez, J. P., \& Rouma, A. C. (2002). El consumo de inhalables y cannabis en la preadolescencia: Análisis multivariado de factores predisponentes. Anales de Psicología, 18(1), 77-93. https://tinyurl.com/y5dpkcgt 
Reiss, S., Peterson, R. A., Gursky, D. M., \& McNally, R. J. (1986). Anxiety sensitivity, anxiety frequency and the prediction of fearfulness. Behaviour Research and Therapy, 24(1), 1-8. https://doi.org/10.1016/0005-7967(86)90143-9

Robles-García, R., Fresán, A., Castellanos-Ryan, N., Conrod, P., Gómez, D., Rafful, C., . . Medina-Mora, M. E. (2014). Spanish version of the Substance Use Risk Profile Scale: factor structure, reliability, and validity in Mexican adolescents. Psychiatry Research, 220(3), 1113 1117. https://doi.org/10.1016/j.psychres.2014.08.057

Rueda, S. (2012). Health inequalities among older adults in Spain: The importance of gender, the socioeconomic development of the region of residence, and social support. Women's Health /ssues, 22(5), e483-e490. https://doi.org/10.1016/j.whi.2012.07.001

Stautz, K., \& Cooper, A. (2013). I mpulsivity-related personality traits and adolescent alcohol use: a meta-analytic review. Clinical Psychology Review, 33(4), 574-592. https://doi.org/10.1016/j.cpr.2013.03.003

Sánchez-Sosa, J. C., Villarreal-González, M. E., Ávila Guerrero, M. E., Vera Jiménez, A., \& Musitu, G. (2014). Contextos de socialización y consumo de drogas ilegales en adolescentes escolarizados. Psychosocial Intervention, 23(1), 69-78. https://doi.org/10.5093/in2014a7

Teichman, M., Barnea, Z., \& Rahav, G. (1989). Sensation seeking, state and trait anxiety, and depressive mood in adolescent substance users. International Journal of the Addictions, 24(2), 87-99. https://doi.org/10.3109/10826088909047277

Woicik, P. A., Stewart, S. H., Pihl, R. O., \& Conrod, P. J. (2009). The Substance Use Risk Profile Scale: A scale measuring traits linked to reinforcement-specific substance use profiles. Addictive Behaviors, 34(12), 1042-1055. https://doi.org/10.1016/j.addbeh.2009.07.001

Zuckerman, M. (1979). Sensation Seeking. Hillsdale, NJ: Lawrence Erlbaum Association.

\section{Para citar en APA}

Mendigutxial-Sorabilla, G. y López-Goñi, J. J. (2020). Comportamientos de riesgo y dimensiones de personalidad en la adolescencia. Terapia Psicologíca (En línea), 38(3), 383-401. https://doi.org/ $10.4067 /$ S0718-48082020000300383 